\title{
Biomarkers of lipid metabolism in patients with juvenile idiopathic arthritis: relationship with subtype and inflammatory activity
}

Wellington Douglas Rocha Rodrigues ( $\nabla$ wellington.nutricao@gmail.com )

Universidade Federal de São Paulo: Universidade Federal de Sao Paulo https://orcid.org/0000-00023000-0314

\section{Roseli Oselka Saccardo Sarni}

Federal University of Sao Paulo: Universidade Federal de Sao Paulo

Fernando Luiz Affonso Fonseca

Universidade Federal de Sao Paulo

Annelyse Araujo

Universidade Federal de São Paulo: Universidade Federal de Sao Paulo

Claudi Arnaldo Len

Universidade Federal de São Paulo: Universidade Federal de Sao Paulo

Maria Teresa Terreri

Universidade Federal de São Paulo: Universidade Federal de Sao Paulo

\section{Research article}

Keywords: Juvenile idiopathic arthritis, Acute-phase proteins, Apolipoproteins, Cholesterol.

Posted Date: November 6th, 2020

DOl: https://doi.org/10.21203/rs.3.rs-102464/v1

License: (c) (i) This work is licensed under a Creative Commons Attribution 4.0 International License.

Read Full License

Version of Record: A version of this preprint was published at Pediatric Rheumatology on May 3rd, 2021. See the published version at https://doi.org/10.1186/s12969-021-00538-w. 


\section{Abstract}

Background: To describe the biomarkers of lipid metabolism in children and adolescents with polyarticular and systemic JIA and to relate them to diseases subtypes, diseases activity markers, and nutritional status.

Methods: A cross-sectional study including 62 JIA patients was performed. The following variables were evaluated: disease activity and medications used, body mass index, height for age (z-score), skin folds (bicipital, tricipital, subscapular and suprailiac), food intake based on three 24-hour food recalls, lipid profile (total cholesterol (CT), low-density lipoprotein (LDL), high-density lipoprotein (HDL), triglycerides (TG) and non-HDL (N-HDLC), glycemia and insulin, erythrocyte sedimentation rate (ESR), ultrasensitive Creactive protein (Us-CRP) and apolipoproteins $A-I$ and $B(A p o ~ A-I$ and Apo B).

Results: Dyslipidemia was observed in $83.3 \%$ of the patients. Based on classical lipid profile, low HDL-c levels was the most frequently alteration observed. Inadequate levels of LDL-C, Apo B and NHDL-c were significantly more frequent in the systemic JIA subtype when compared to the polyarticular subtype $(p=$ $0.017,0.001$ and 0.042 respectively). Patients on biological therapy had a better adequacy of Apo A-I concentrations. The ESR showed a negative correlation with Apo A-l level $(r=-0.25, p=0.047)$.

Conclusion: We concluded that dyslipidemia is common in patients with JIA, especially in systemic subtype. The systemic subtype and an elevated ESR were associated with lower concentrations of Apo AI, suggesting the participation of the inflammatory process.

\section{Background}

Juvenile idiopathic arthritis (JIA) is the most common chronic rheumatic disease in the pediatric population $^{[1,2]]}$. JIA is defined as chronic inflammation in one or more joints for a minimum period of six weeks in children aged 16 years or younger ${ }^{[3]}$.

Advances in the diagnosis and treatment of JIA over the last few decades have modified outcomes in children and adolescents, resulting in a decrease in mortality due to disease activity and secondary infectious complications. However, this group of patients coexists chronically with the disease, and they present early negative outcomes, such as osteoporosis and cardiovascular diseases later on ${ }^{[4]}$.

Studies have shown that adult patients with rheumatoid arthritis have an increased risk for atherosclerotic disease ${ }^{[5,6]}$. In this group, the risk for cardiovascular diseases is 30 to $60 \%$ higher than that in the general population ${ }^{[7]}$. Long-term JIA adult patients in remission might have subclinical signs of inflammation and cardiovascular risk, showed by an increase in the levels of inflammatory cytokines, endothelial activation and oxidative stress markers and adipokines, molecules involved in the alteration of the vascular system ${ }^{[8]}$. 
The identification of cardiovascular risk in the pediatric age group is a major challenge, especially in patients with chronic conditions such as $\mathrm{JIA}^{[9]}$. Measurements of the medial-intimal thickness (MIE) and biomarkers of lipid metabolism have been proposed as mechanisms for achieving this ${ }^{[10-13]}$. In adult patients with rheumatoid arthritis, changes in apolipoprotein $B(A p o B)$ concentrations and the Apo B/apolipoprotein A-I (Apo A-I) ratio compared to those in the classical lipid profile have been independently associated with cardiovascular risk ${ }^{[14]}$.

To our knowledge, there are no studies in the literature on children and adolescents with JIA involving lipid biomarkers such as Apo A-I and Apo B that consider the disease onset subtypes.

Thus, we aimed to describe the biomarkers of lipid metabolism in children and adolescents with polyarticular and systemic JIA and to relate them to disease subtypes, disease activity markers and nutritional status.

\section{Patients And Methods}

In this cross-sectional study, at our pediatric rheumatology outpatient clinic, we included 68 consecutive children and adolescents of both genders who were age 5 to 19 years and who had polyarticular or systemic onset JIA according to the International League of Associations for Rheumatology (ILAR) ${ }^{[15]}$.

Patients who did not want to participate or who had insufficient data on disease onset were excluded. Therefore, the sample included 62 patients.

This study was approved by the local ethics and research committee, and informed consent and assent were obtained from the caregivers or caregivers and patients.

Data on the demographic, clinical and laboratory findings were obtained, and a nutritional, food intake and family cardiovascular risk assessment was performed. A blood sample was collected on the same day.

Active disease was diagnosed according to the Wallace criteria, and the patients were classified as inactive, in remission on medication, in remission off medication and active ${ }^{[16]}$.

The nutritional assessment was performed based on the body mass index (BMI) and height for age $(\mathrm{H} / \mathrm{A})$, and patients were classified according to World Health Organization data. Waist circumference was classified as increased when it was higher than the 90th percentile according to Freedman et al. ${ }^{[17]}$. Arm circumference, triceps and subscapular skinfolds were assessed and classified according to Frisancho ${ }^{[18]}$. Pubertal stage assessment was performed according to Marshall \& Tanner, considering breast development $(M)$ for girls and genitalia $(G)$ for boys.

The food intake assessment was performed through three 24 -hour food recalls ${ }^{[19]}$. To calculate the intake of macronutrients, total fat, cholesterol, monounsaturated, polyunsaturated, saturated and trans fats, 
fiber and sodium, the Nutrition Support Program (Icalcdiet/Unifesp) was used, based on American and Brazilian references.

After a 12-h fast, blood was collected for analysis of the following lipid metabolism biomarkers: total cholesterol (TC), lipid fractions [very-low-density lipoprotein cholesterol (VLDL-C), LDL-c, high-density lipoprotein cholesterol (HDL-c)], triglycerides (TG), Apo A-I and Apo B, insulin, glucose, and ultra-sensitive C-reactive protein (us-CRP). Insulin and us-CRP were determined using chemiluminescence and immunoturbidimetric methods, respectively.

Apo A-I and Apo B were measured using enzyme-linked immunosorbent assay (ELISA) PRO kits for human Apo A-I and Apo B (Mabtech, Cincinnati, OH, USA) and also evaluated glycemia by enzymatic colorimetric assay and erythrocyte sedimentation rate (ESR).

The HOMA-IR (homeostasis model assessment of insulin resistance) value, classified as increased when $>3.16^{[20]}$. To classify the lipid profile, the cutoff points proposed by the American Academy of Pediatrics were adopted $^{[21]}$. Values of non-HDL (NHDL-c) were classified according to the Bogalusa Heart Study ${ }^{[22]}$. The ratios of TC/HDL-c, LDL-c/HDL-c, Apo B/Apo A-I, TG/HDL, and LDL-c/Apo B were also calculated.

For the qualitative variables, Pearson's Chi-square association test or Fisher's exact test was used, and for the quantitative variables, the Mann-Whitney test was used. We used the median as a measure of central tendency and the interquartile range (IQ) (25-75\%) as a measure of dispersion. To evaluate the correlations between cardiovascular risk factors and HDL-C and Apo A-I, the Spearman correlation test was performed. The level of significance was set at $p<0.05$.

\section{Results}

Table 1 shows demographic, clinical and food intake data of children and adolescents with systemic and polyarticular JIA subtypes. The median BMI was 17.9 (16.1-21.0), 3 patients (4.8\%) were underweight and $13(21 \%)$ were overweight. Regarding the other anthropometric data, the patients had medians of $-0.29(-1.1-0.5)$ for Z-score height/age, $65 \mathrm{~cm}(58-71 \mathrm{~cm})$ for waist circumference, $0.43(0.40-0.46)$ for waist/height, $22 \mathrm{~cm}(19-24.7 \mathrm{~cm})$ for arm circumference, $13 \mathrm{~mm}(9-18 \mathrm{~mm})$ for triceps skinfold, $9 \mathrm{~mm}$ (6-13 mm) for subscapular skinfold and a 20.8\% (15.4-26.7\%) percentage of body fat. Regarding food intake, the patients presented a median of $1933.6 \mathrm{kcal}(1795-2177 \mathrm{kcal})$ for total energy intake. None of the patients presented with a carbohydrate intake higher than recommended, although 3 (4.9\%) presented with a protein intake higher than the recommendation, and 18 (29\%) presented with a lipid intake higher than the recommendation. The patients had medians of $24.4 \mathrm{~g}(20.9-30.9 \mathrm{~g})$ for saturated fat and $4.2 \mathrm{~g}$ (2.2-6.4 g) for trans-fat; $47(75.8 \%)$ presented a saturated fat intake higher than the recommendation, and $49(79 \%)$ presented a trans-fat intake higher than the recommendation. 
Table 1

Demographic, clinical and food intake data of children and adolescents with polyarticular and systemic JIA subtypes.

\begin{tabular}{|c|c|}
\hline Variables & $N=62$ \\
\hline Female N (\%) & $46(74.2)$ \\
\hline Age, Mean \pm SD & $7.7 \pm 4.3$ \\
\hline \multicolumn{2}{|l|}{ Socio-economic classification N(\%) } \\
\hline A & $5(0.1)$ \\
\hline B & $15(24.2)$ \\
\hline C & 39 (62.9) \\
\hline$D / E$ & $3(4.8)$ \\
\hline \multicolumn{2}{|l|}{ Pubertal stage N(\%) } \\
\hline Pre-pubertal & $20(32.3)$ \\
\hline Pubertal & $26(41.9)$ \\
\hline Pos-pubertal & $16(25.8)$ \\
\hline \multicolumn{2}{|l|}{ Disease subtype N(\%) } \\
\hline JIA RF+ & $10(16.1)$ \\
\hline JIA RF- & $39(62.9)$ \\
\hline Systemic & $13(21.0)$ \\
\hline Disease follow up time (years), Mean \pm SD & $5.0 \pm 3.4$ \\
\hline \multicolumn{2}{|l|}{ Disease activity $\mathrm{N}(\%)$} \\
\hline Active & $21(33.9)$ \\
\hline Inactive & $5(8.0)$ \\
\hline Remission on medication & $29(46.8)$ \\
\hline Remission off medication & $7(11.3)$ \\
\hline Biological DMARDs N(\%) & $16(25.8)$ \\
\hline Synthetic DMARDs N(\%) & $40(64.5)$ \\
\hline Corticosteroids N(\%) & $6(9.7)$ \\
\hline
\end{tabular}

CHAQ - Childhood Health Assessment Questionnaire; JIA - juvenile idiopathic arthritis, DMARDs disease modified antirrheumatic drugs 


\begin{tabular}{|lll|}
\hline Variables & $\mathrm{N}=62$ & \\
\hline Cumulative dosis of corticosteroids (mg) & 2620 & $1762.5-7483.7$ \\
\hline CHAQ altered N(\%) & $17(27.4)$ & \\
\hline CHAQ, Mean \pm SD & $1.2(0.8)$ & \\
\hline Food intake & & $1795.0-2177.0$ \\
\hline Total energy intake (kcal) & 1933.6 & $227.0-281.2$ \\
\hline Carbohydrate (g) & 257.9 & \\
\hline Above recommendation & 0 & $68.0-94.5$ \\
\hline Proteins (g) & 83.6 & $56.4-78.4$ \\
\hline Above recommendation & $3(4.9)$ & \\
\hline Lipids (g) & 67.2 & $20.9-30.9$ \\
\hline Above recommendation & $18(29)$ & \\
\hline Saturated fats (g) & 24.4 & \\
\hline Above recommendation & $47(75.8)$ & \\
\hline Monounsaturated fats (g) & 23.8 & $19.3-27.8$ \\
\hline Above recommendation & $1(1.6)$ & \\
\hline Polyunsaturated fats (g) & 10.8 & \\
\hline Above recommendation & 0 & \\
\hline Trans fat (g) & 4.2 & \\
\hline Above recommendation & $49(79)$ & \\
\hline Total cholesterol(mg) & 224.1 & \\
\hline Above recommendation & $18(29)$ & \\
\hline CHAQ - Childhood Health Assessment Questionnaire; JIA - juvenile idiopathic arthritis, DMARDs - & & \\
\hline disease modified antirrheumatic drugs & & \\
\hline
\end{tabular}

Table 2 shows body mass index, disease activity and laboratory and lipid profile findings in children and adolescents with juvenile idiopathic arthritis according to HDL-c level. 
Table 2

Body mass index, disease activity and laboratory and lipid profile findings in children and adolescents with juvenile idiopathic arthritis according to HDL-c level

\begin{tabular}{|c|c|c|c|}
\hline \multirow[t]{2}{*}{ Variables } & \multicolumn{3}{|l|}{ HDL-C } \\
\hline & Adequate & Borderline-low & $P$ \\
\hline & $n=42$ & $n=20$ & \\
\hline Pubescent, N (\%) & $33(78.6)$ & $9(45)$ & 0.559 \\
\hline BMI & $17.9(17-21.2)$ & $18.6(16.1-20.3)$ & 0.952 \\
\hline Z-Score BMI/age > 1 & 7 (16.7) & $6(30)$ & 0.228 \\
\hline CHAQ & $0.27(0.1-0.6)$ & $0.55(0.3-1.03)$ & 0.292 \\
\hline$>0, \mathrm{~N}(\%)$ & $10(23.8)$ & $7(35)$ & 0.356 \\
\hline \multicolumn{4}{|l|}{ Disease activity } \\
\hline Active, N (\%) & $16(38.1)$ & $5(25)$ & 0.308 \\
\hline us-CRP & $1.2(0.5-4.9)$ & $2.8(0.9-15)$ & 0.312 \\
\hline$\geq 3$ & $14(33.3)$ & $10(50.0)$ & 0.208 \\
\hline ESR & $6.5(5.0-30.0)$ & $19.5(6.0-32.0)$ & 0.191 \\
\hline$\geq 20$ & $15(35.7)$ & $10(50.0)$ & 0.407 \\
\hline LDL-C & $86.4(72.2-103.0)$ & $84.4(71.2-99.4)$ & 0.646 \\
\hline Borderline/High & $8(19.0)$ & $4(20.0)$ & 1.000 \\
\hline Triglycerides & $66.5(51.0-85.0)$ & $62.0(51.5-88.5)$ & 0.821 \\
\hline Borderline/High & $12(28.6)$ & $5(25.0)$ & 1.000 \\
\hline Apo A-1 & $118.0(108.0-137.0)$ & $100.0(92.0-106.5)$ & $<0.001$ \\
\hline Borderline/Low & $23(54.8)$ & $19(95.0)$ & 0.001 \\
\hline Apo B & $72.5(58.0-84.0)$ & $68.0(58.5-83.5)$ & 0.821 \\
\hline Borderline/Low & $5(11.9)$ & $4(20.0)$ & 0.453 \\
\hline NHDL-C & $99.0(87.0-124.0)$ & $96.5(82.5-119.5)$ & 0.474 \\
\hline
\end{tabular}

Tests: Fisher's exact or Chi-square test, or Pearson or Mann-Whitney, $\mathrm{p}<0.05$; median (IQ: 25-75); BMI- body mass index; CHAQ- Childhood Health Assessment Questionnaire; us-CRP- ultra-sensitive C reactive protein; ESR- erythrocyte sedimentation rate; LDL-C- low-density lipoprotein cholesterol; HDLc- high-density lipoprotein cholesterol; NHDL- non-high-density lipoprotein cholesterol; Apo A-Iapolipoprotein A-1; Apo B-apolipoprotein B. 


\begin{tabular}{|l|lc|}
\hline \multicolumn{1}{|c|}{ Variables } & HDL-c \\
\hline Borderline/Low & $12(28.6)$ & $5(25.0)$ \\
\hline
\end{tabular}

Dyslipidemia was observed in $83.3 \%$ of the patients when altered lipid biomarkers (CT, LDL-C, HDL-C, TG, NHDL-C, Apo A-I and Apo B) were considered. Borderline and low levels of HDL was the most frequent dyslipidemia based on classical lipid profile (20 patients - 32.3\%). Decrease in Apo A-I level was present in 42 patients $(67.7 \%)$ and more frequent in patients who did not use biological agents (83\% versus $16 \%$ ). The frequency of borderline /high values of LDL-c was statistically higher in systemic JIA than in polyarticular JIA $(p=0.017)$. In addition, we observed a higher frequency of Apo B and NHDL-c alterations and higher values of Apo B/Apo A-1 ratio, CT/HDL-c ratio and LDL-C/HDL-c ratio in systemic JIA.

Regarding the HDL-c variable, we observed that patients with borderline /low lipoprotein had lower levels and a higher frequency of inadequate Apo A-I. We also observed that the familial cardiovascular risk was present in 42 patients (67\%).

We did not find association of inadequate Apo A-I or HDL-c with time of duration and disease subtype, use of medication (glucocorticoid), and with score-z BMI. The association of Apo A-I with disease activity showed a value of $p=0.055$. We also observed that patients off biological agents had a higher frequency of inadequate Apo A-I than patients on biological agents ( $76 \%$ vs. $43.8 \%$, respectively), $(p=0.017)$. The Apo-A1 levels were not associated with laboratory findings, classic lipid profile, Apo B or glucose levels.

The frequency of borderline / high LDL-c values was statistically higher in systemic JIA than in polyarticular JIA ( $p=0.017)$. In addition, we also observed statistically higher values of Apo $B, A p o B$ / Apo A-1 ratio, non-HDL-c, CT / HDL-c ratio and LDL-c / HDL-c in systemic JIA ( $p=0.03,0.042,0.043$ and 0.037 , respectively).

We did not observe a correlation between CRP and the variables related to the lipid profile (Spearman's correlation analysis). However, the ESR showed a negative correlation with Apo A-l levels $(r=-0.25, p=$ 0.047) (Fig. 1).

We also observed a positive correlation between the cumulative glucocorticoid dose and TC $(r=0.58, p=$ 0.018), LDL-c $(r=0.64, p=0.008)$ and NHDL-c $(r=0.64 ; p=0.007)$.

\section{Discussion}

The present study showed that dyslipidemia affected more than three-quarters of JIA patients, with predominance of borderline-low HDL-c based on classical lipid profile. Similar results were found in a 
retrospective study published by our research group ${ }^{[23]}$. We observed a higher frequency of increased LDL-c, NHDL-c and Apo B in the systemic subtype compared to the polyarticular subtype. Systemic-onset JIA and elevated ESR were associated with lower concentrations of Apo A-I, suggesting the involvement of the inflammatory process.

Studies evaluating non-classical biochemical markers related to lipid metabolism in children and adolescents with autoimmune rheumatic diseases are scarce ${ }^{[12,22,24-26]}$.

The mechanisms involved in the pathogenesis of dyslipidemia and cardiovascular risk in patients with JIA have not yet been fully elucidated. Inflammation with endothelial dysfunction and increased levels of proinflammatory cytokines have been described ${ }^{[27]}$. In addition, inadequate food intake, physical inactivity, overweight and the use of certain medications, such as glucocorticoids, also aggravate dyslipidemia ${ }^{[28]}$. The use of glucocorticoids seems to play an important role in dyslipidemia, considering that, in our study, we observed an association between a higher cumulative dose and alterations in TC, LDL-C and NHDL-C.

In patients with rheumatoid arthritis, the prevalence of dyslipidemia varies between 55 and $65 \%$ [29]. In our sample, the prevalence was $82.3 \%$ when we considered changes in Apo A-I and Apo B. Although these apolipoproteins are considered good biomarkers for cardiovascular risk, several studies did not consider them in the evaluation of dyslipidemia $[24,30,31]$.

Anthropometric markers of adiposity, such as BMI, waist circumference and intake of fats and carbohydrates, were normal for most patients. This fact could be explained by the role of the multiprofessional team in our service and suggests important participation of the disease and its treatment in the pathogenesis of dyslipidemia.

Although glucocorticoids were used in only about $10 \%$ of our patients, we observed an association between the cumulative dose and dyslipidemia. Marangoni et al. reported a trend of association between glucocorticoid use and increased levels of LDL-c ${ }^{[30]}$. They also pointed out that the decrease in HDL-c level was not associated with the use of glucocorticoid medication. The progressively increasing use of synthetic DMARDs and biological agents minimizes the harmful effects of glucocorticoids on lipid metabolism [32].

We know that systemic-onset JIA is associated with greater laboratory and inflammatory alterations, such as anemia, increased platelet count and acute phase proteins and frequent use of corticosteroids. This explains the major changes in LDL-C, Apo B and NHDL-c found in our patients with this JIA subtype. In a longitudinal study, Yeh et al. evaluated the lipid profile and atherogenic index of JIA patients after treatment with etanercept and found higher concentrations of HDL-C and lower TG and TC/HDL in the group they called responders (with inactive disease) compared to non responders ${ }^{[33]}$. The non responder group, which basically consisted of patients with the systemic subtype, did not show improvement in the 
lipid profile. Other studies addressing dyslipidemia in JIA patients did not individualize the different subtypes and did not evaluate biomarkers such as Apo A-I and Apo B ${ }^{[24,31]}$.

Although an adequate HDL-c level was not associated with demographic and clinical characteristics, nutritional status or food intake, patients with an adequate HDL-c had a higher value and higher frequency of normal levels of Apo A-I. In the literature, it is well-established that adequate levels of HDL-C, due to its antioxidant (mainly by the presence of Apo A-I), anti-inflammatory, antiatherogenic, antithrombotic functions and cholesterol transportation, are related to a reduction in cardiovascular risk $[34,35]$. However, it is worth mentioning that, in the presence of systemic inflammation, there may be a conversion of protective HDL-c to proinflammatory HDL-C, and the reduction in the production of Apo A-I is one of the proposed mechanisms of this transformation ${ }^{[34,35]}$.

Studies have shown an association between disease activity and dyslipidemia ${ }^{[33,36]}$. Although we have a larger sample compared to other studies ${ }^{[33,36]}$, we found only a tendency towards an association between JIA activity and altered levels of Apo A-I, that is, patients with active disease had a higher frequency of reduction in Apo A-I than patients with inactive disease (in remission or not).

The decrease in Apo A-I level was more frequent in patients who did not use biological agents (83\% versus $16 \%$ ). This can be explained by the action of these agents inhibiting the production of cytokines and, consequently, inflammation, thus improving the lipid profile.

Rodriguez-Jimenez showed that tumor necrosis factor-a (TNF-a) is associated with increased cardiovascular risk, increased hepatic CRP synthesis and decreased HDL-c levels ${ }^{[37]}$. Under normal conditions, the major protein fraction of HDL is Apo A-I. However, the literature has shown that, in the presence of inflammation, mainly in increased interleukin-1 and TNF-a conditions, there is an increase in the production of serum amyloid A (SAA) by hepatocytes ${ }^{[38,39]}$. This protein, on the other hand, has atherogenic action, and when released into the bloodstream rapidly, it associates with the third fraction of HDL (HDL3), decreasing serum concentrations of Apo A-I. A study in patients with JIA has shown that anti-TNF-a therapy alters the proatherogenic lipid profile of these patients ${ }^{[40]}$.

Interestingly, a negative correlation between the ESR and Apo A-I concentration was observed. We did not find any studies in the literature in adults or children with chronic arthritis that correlated inflammatory markers with lipid biomarkers. Although other inflammatory markers, such as proinflammatory cytokines,

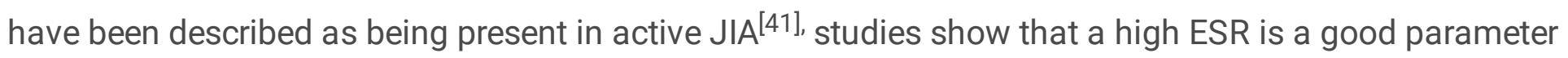
of disease activity ${ }^{[42]}$, and when it is normal, it is one of the variables included in Wallace's inactivity criteria ${ }^{[15]}$. Based on this, our findings suggest that, in the presence of high disease activity and/or a high ESR, the investigation of lipid metabolism biomarkers should be expanded.

This study has some limitations, such as the absence of a control group and the lack of evaluation of the practice of physical activity by the patients. In addition, the sample size may have been insufficient to 
show significant differences between the various variables. However, this study is relevant and original because it is the first study on dyslipidemia showing differences between the JIA subtypes.

\section{Conclusion}

We conclude that dyslipidemia is frequently presented in patients with $\mathrm{JIA}$, although the majority of patients presented a satisfactory nutritional status and absence of metabolic syndrome. However, inadequate consumption of atherogenic lipids occurred frequently. Another important point was the association between the presence of systemic JIA and an elevated ESR with a higher frequency of an altered lipid profile. We also observed that the use of biological agents may be a protective factor for dyslipidemia.

Therefore, future studies should involve, in addition to lipid biomarkers such as Apo A-I and Apo B, the evaluation of biomarkers of inflammation (cytokines), direct markers of vascular injury and the performance of imaging methods to measure atherosclerotic risk, in order to understand the cardiovascular risk in these patients and thus be able to outline strategies and interventions to reduce this risk.

\section{List Of Abbreviations}

JIA - Juvenile idiopathic arthritis

MIE - medial-intimal thickness

Apo A-I - apolipoprotein A-I

Apo B - apolipoprotein B

ILAR - International League of Associations for Rheumatology

BMI - body mass index

$H \backslash A$ - height for age

UNIFESP - Universidade Federal de São Paulo

TC - total cholesterol

VLDL - lipid fractions [very-low-density lipoprotein cholesterol

LDL-c - low-density lipoprotein cholesterol

HDL-c - high-density lipoprotein cholesterol

TG - triglycerides 
us-CRP - ultra-sensitive C-reactive protein (us-CRP)

ESR - erythrocyte sedimentation rate

HOMA-IR - homeostasis model assessment of insulin resistance

NHDL-c - non high-density lipoprotein cholesterol

IQ - interquartile range

DMARD - Disease-modifying antirheumatic drugs

TNF-a - tumor necrosis factor-a

SAA - serum amyloid A

\section{Declarations}

Ethics approval and consent to participate - This study was approved by the local ethics and research committee, and informed consent and assent were obtained from the caregivers or caregivers and patients (Ethics and Research Committee of Unifesp, under protocol number 1.415.583)

Consent for publication - Not applicable

Availability of data and material - The datasets generated during and/or analysed during the current study are not publicly available due [REASON WHY DATA ARE NOT PUBLIC] but are available from the corresponding author on reasonable request.

Competing interests: The authors declare that they have no competing interests

Funding - Not applicable (own financing)

\section{Authors' contributions}

W.D.R.R - Participated in its design and helped to draft, analyzed and interpreted the patient's data and contributed to the writing of the manuscript.

R.O.S.S - Analyzed and interpreted patient data and contributed to the writing of the manuscript.

F.L.A.F - Participated in the determination and analysis of biochemical tests.

A.A - Participated in the editing of the manuscript and contributes to the writing

C.A.L - Participated in the editing of the manuscript and contributes to the writing

M.T.T - Participated in its design and coordination and helped to draft the manuscript 
$\star \star \star$ All authors read and approved the final manuscript

Acknowledgement: Coordenação de Aperfeiçoamento de Pessoal de Nível Superior (CAPES) for the scholarship award.

\section{References}

1. Silva VBM, Nicacio AGF, Lederman HRR, Hilário MOE, Terreri MTSLRA. Association between the ultrasonographic and clinical findings in the hips of patients with juvenile idiopathic arthritis. Rev Bras Reumatol. 2013;53:322-327.

2. Breda L, Di Marzio D, Giannini C, et al. Relationship between inflammatory markers, oxidantantioxidant status and intima-media thickness in prepubertal children with juvenile idiopathic arthritis. Clin Res Cardiol. 2013;102:63-71.

3. Yamashita E, Terreri MTRA, Hilário MOE, Len CA. Prevalence of juvenile idiopathic arthritis in children aged 6 to 12 years in Embu das Artes, state of São Paulo, Brazil. Rev Bras Reumatol. 2013;53:542545 .

4. Hashkes PJ, Wright BM, Lauer MS, et al. Mortality outcomes in pediatric rheumatology in the US. Arthritis Rheum. 2010;62:599-608.

5. El-Banna H, Jiman-Fatani A. Anti-cyclic citrullinated peptide antibodies and paraoxonase-1 polymorphism in rheumatoid arthritis. BMC Musculoskelet Disord. 2014;15:1-7.

6. Charles-Schoeman C, Lee YY, Shahbazian A, et al. Association of paraoxonase 1 gene polymorphism and enzyme activity with carotid plaque in rheumatoid arthritis. HHS Public Access. 2015;65:27652772.

7. Naranjo A, Sokka T, Descalzo MA, et al. Cardiovascular disease in patients with rheumatoid arthritis: results from the QUEST-RA study. Arthritis Res Ther. 2008;10:1-10.

8. Aranda-Valera, I.C., Arias de la Rosa, I., Roldán-Molina, R. et al. Subclinical cardiovascular risk signs in adults with juvenile idiopathic arthritis in sustained remission. Pediatr Rheumatol. 2020;18; 1-12.

9. Barsalou J, Bradley TJ, Silverman ED. Cardiovascular risk in pediatric-onset rheumatological diseases. Arthritis Res Ther. 2013;15:1-12.

10. Głowińska-Olszewska B, Bossowski A, Dobreńko E, et al. Subclinical cardiovascular system changes in obese patients with juvenile Idiopathic Arthritis. Mediators Inflamm. 2013;13:1-11.

11. Vlahos AP, Theocharis $P$, Bechlioulis $A$, et al. Changes in vascular function and structure in juvenile idiopathic arthritis. Arthritis Care Res. 2011;63:1736-1744.

12. Skare TL, Silva MB, Negreiros P. Lipid profile in adult patients with idiopathic juvenile arthritis. Rev Bras Reumatol. 2013;53:371-374.

13. Jednacz E, Rutkowska-Sak L. Atherosclerosis in juvenile idiopathic arthritis. Mediators Inflamm. 2012;12:1-5. 
14. Mackey RH, Kuller LH, Moreland LW. Cardiovascular disease risk in patients with rheumatic diseases. Clin Geriatr Med. 2017;33:105-117.

15. Petty R, Southwood T, Manners P. International League of Associations for Rheumatology. International League of Associations for Rheumatology classification of juvenile idiopathic arthritis. J Rheumatol. 2004;2:390-392.

16. Wallace CA, Ruperto N, Giannini E, Childhood Arthritis and Rheumatology Research Alliance, Pediatric Rheumatology International Trials Organization, Pediatric Rheumatology Collaborative Study Group. Preliminary criteria for clinical remission for select categories of juvenile idiopathic arthritis. J Rheumatol. 2004; 31:2290-2294.

17. Freedman DS, Serdula MK, Srinivasan SR, Berenson GS. Relation of circumferences and skinfold thicknesses to lipid and insulin concentrations in children and adolescents: The Bogalusa Heart Study. Am J Clin Nutr. 1999;69:308-317.

18. Frisancho AR. Triceps skin fold and upper arm muscle size norms for assessment of nutritional status. Am J Clin Nutr. 1974;27:1052-1058.

19. Fisberg RM, Marchioni DML, Colucci ACA. Assessment of food consumption and nutrient intake in clinical practice. Arq Bras Endocrinol Metabol. 2009;53:617-624.

20. Keskin M, Kurtoglu S, Kendirci M, Atabek ME, Yazici C. Homeostasis model assessment is more reliable than the fasting glucose/insulin ratio and quantitative insulin sensitivity check index for assessing Insulin resistance among obese children and adolescents. Pediatrics. 2005;115: 500-503.

21. American Academy Pediatrics. Expert panel on integrated guidelines for cardiovascular health and risk reduction in children and adolescents: Summary report. Pediatrics.2011;128:213-256.

22. Srinivasan SR,Frontini MG,Xu J, Berenson GS. Utility of childhood non-high-density lipoprotein cholesterol levels in predicting adult dyslipidemia and other cardiovascular risks: The Bogalusa Heart Study. Pediatrics. 2006;118:201-206.

23. Rodrigues WDR, Sarni ROS, Abad TTO, da Silva SGL, de Souza FIS, Len CA, et al. Lipid profile of pediatric patients with chronic rheumatic diseases - a retrospective analysis. Rev Assoc Med Bras. 2020; 66:1093-1099.

24. Pugliese C, van der Vinne RTA, Campos LMA, et al. Juvenile idiopathic arthritis activity and function ability: deleterious effects in periodontal disease? Clin Rheumatol. 2016;35(1):81-91.

25. Ortiz TT, Terreri MT, Caetano M, et al. Dyslipidemia in pediatric systemic lupus erythematosus: The relationship with disease activity and plasma homocysteine and cysteine concentrations. Ann Nutr Metab.2013;63:77-82.

26. Machado D, Sarni ROS, Abad TTO, et al. Lipid profile among girls with systemic lupus erythematosus. Rheumatol Int. 2017;37:43-48.

27. Coulson EJ, Ng W-F, Goff I, Foster HE. Cardiovascular risk in juvenile idiopathic arthritis. Rheumatology. 2013;52:1163-1171.

28. Caetano MC, Ortiz TT, Terreri MTSLRA, et al. Inadequate dietary intake of children and adolescents with juvenile idiopathic arthritis and systemic lupus erythematosus. J Pediatr. 2009;85:509-515. 
29. Toms TE, Panoulas VF, Kitas GD. Dyslipidaemia in rheumatological autoimmune diseases. Open Cardiovasc Med J. 2011;5:64-75.

30. Marangoni RG, Hayata AL, Borba EF, Azevedo PM, Bonfa E, Schainberg GC. Decreased high-density lipoprotein cholesterol levels in polyarticular juvenile idiopathic arthritis. Clinics. 2011;66:1549-1552.

31. Bohr AH, Pederson KF, Nilsen CH, Muller KG. Lipoprotein cholesterol fractions are related to markers of inflammation in children and adolescents with juvenile idiopathic arthritis: a cross sectional study. Ped Rheumatol. 2016;14:2-9.

32. Mani $\mathrm{P}$, Uno $\mathrm{K}$, Duong $\mathrm{M}$, et al. HDL function and subclinical atherosclerosis in juvenile idiopathic arthritis. Cardiovasc Diagn Ther. 2016;6:34-43.

33. Yeh KW, Lee CM, Chang CJ, Lin YJ, Huang JL. Lipid profiles alter from pro-atherogenic into less atherogenic and proinflammatory in juvenile idiopathic arthritis patients responding to anti TNF-a treatment. PLoS One. 2014;9:1-7.

34. Hong F, Xa L. Dysfunctional high-density lipoprotein. Curr Opin Endocrinol Diabetes Obes. 2009;12:156-162.

35. Benatti FB, Miossi R, Passareli $M$, et al. The effects of exercise on lipid profile in systemic lupus erythematosus and healthy individuals: A randomized trial. Rheumatol Int. 2015;35:61-69.

36. Tselepis AD, Elisaf M, Besis S, Karabina SAP, John Chapman M, Siamopoulou A. Association of the inflammatory state in active juvenile rheumatoid arthritis with hypo-high-density lipoproteinemia and reduced lipoprotein- associated platelet-activating factor acetylhydrolase activity. Arthritis Rheum. 1999;42:373-383.

37. Rodriguez-Jimenez NA, Garcia-Gonzalez CE, Ayala-Lopez KP, et al. Modifications in lipid levels are independent of serum TNF-a in rheumatoid arthritis: results of an observational 24-week cohort study comparing patients receiving etanercept plus methotrexate or methotrexate as monotherapy. Biomed Res Int. 2014;41:1-11.

38. Raicheil EMV, Andre DN, Freitas A, Munhoz E, Delongui F. The laboratorial tests and its importance in the clinical course of therapeutic monitoring in Graves' disease and rheumatoid arthritis. Rev Bras Anal Clin. 2004;36:201-206.

39. Gayer CRM, Pinheiro GRC, Andrade CAF, Freire SM, Coelho MGP. Evaluation of the serum amyloid A protein in the rheumatoid arthritis clinical activity. Rev Bras Reumatol. 2003;43:199-205.

40. De Sanctis S, Marcovecchio ML, Gaspari S, et al. Etanercept improves lipid profile and oxidative stress measures in patients with juvenile idiopathic arthritis. J Rheumatol. 2013;40:943-948.

41. Lin YT, Wang CT, Gershwin ME, Chiang BL. The pathogenesis of oligoarticular/polyarticular vs systemic juvenile idiopathic arthritis. Autoimmunity Reviews. 2011;10:482-489.

42. Nicácio AAMF, Damazio KC, Terreri MT, Rassi IE, Hilário MOE. Acute phase reactants evaluation in children and adolescents with juvenile idiopathic arthritis and its correlation with disease activity. Rev Paul Pediatr. 2009;27:193-197.

\section{Figures}




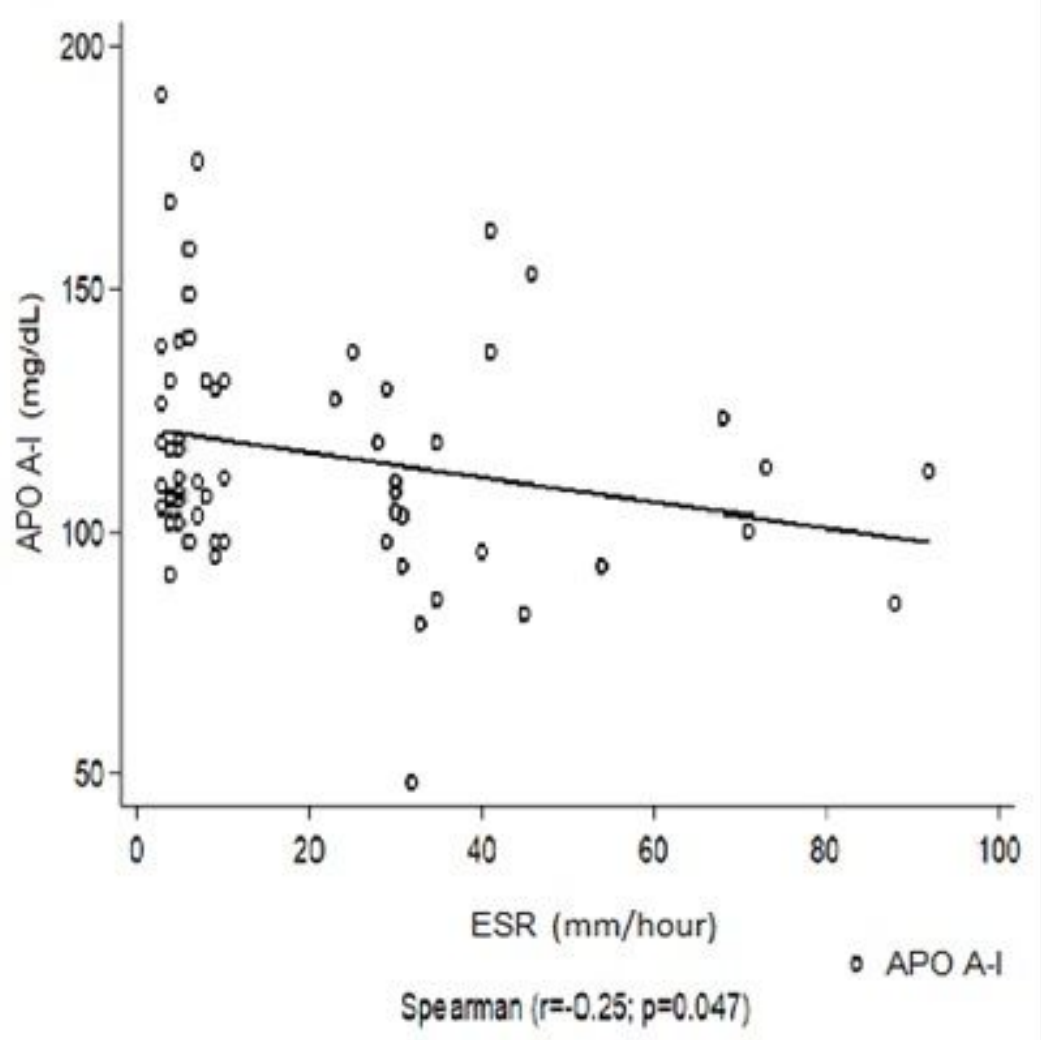

Figure 1

Correlation between levels of erythrocyte sedimentation rate and Apolipoprotein A-l in children and adolescents with juvenile idiopathic arthritis

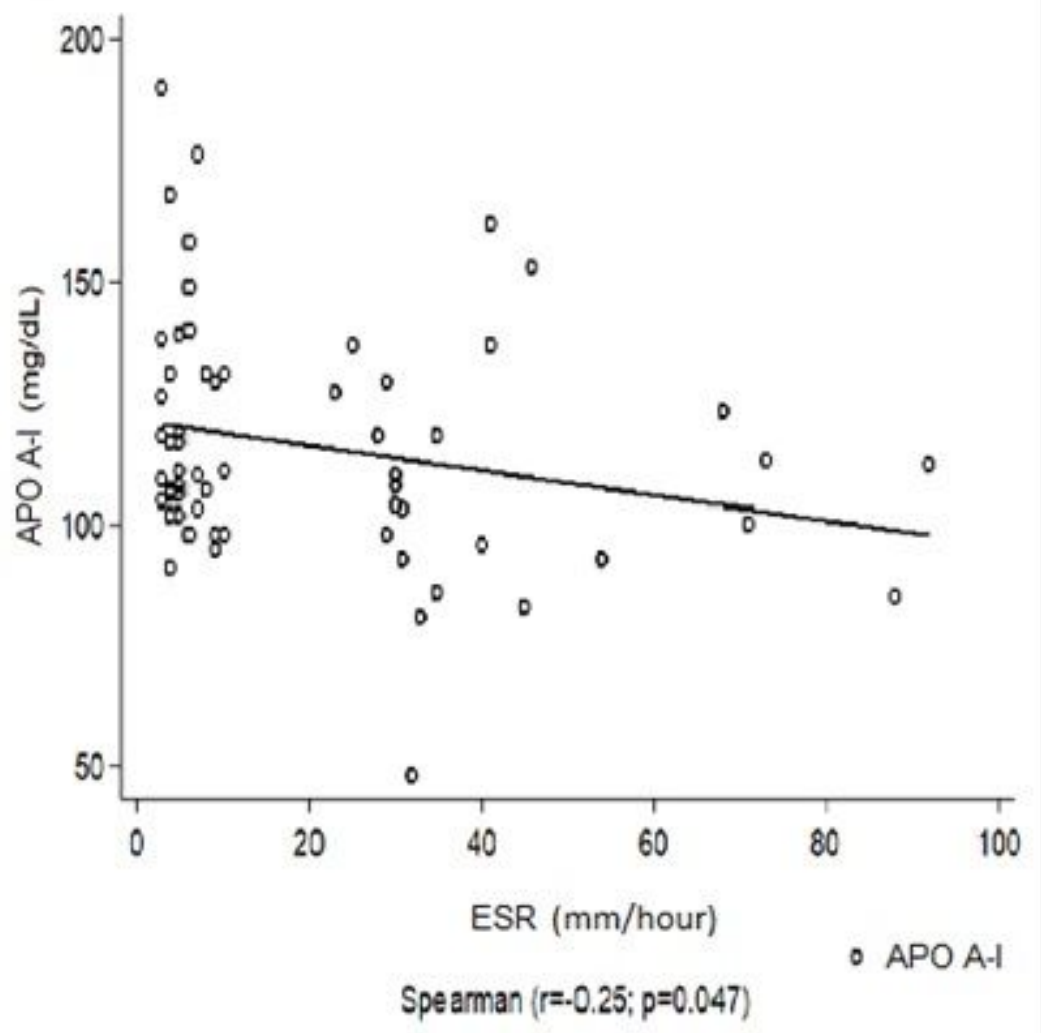

Figure 1 
Correlation between levels of erythrocyte sedimentation rate and Apolipoprotein A-I in children and adolescents with juvenile idiopathic arthritis 